\title{
Effect of Doxycycline on Aseptic Reperfusion Injury in Ovarian Torsion
}

\author{
Gizem BEKTAS', Yetkin KARASU1, Engin YURTCU², Cihan TOGRUL², Sebnem OZYER SEN², \\ Aylin ONDER DIRICAN², Ozlem UZUNLAR ${ }^{2}$, Tayfun GUNGOR ${ }^{2}$, Muzaffer CAYDERE ${ }^{3}$
}

Ankara, Turkey

\section{ABSTRACT}

OBJECTIVE: Reperfusion injury occurs when the condition causing ischemia in ovarian torsion is corrected and the blood supply is re-established. The aim of this study is to evaluate whether doxycycline treatment reduces reperfusion injury in a rat model.

STUDY DESIGN: Thirty-five female albino Wistar rats were split into the five groups. Sham: Sham operation; ischemia (I): 2 hours of ischemia; ischemia and reperfusion (I/R): 2 hours of ischemia followed by 2 hours of reperfusion; Sham-Dc: Sham operation and doxycycline $10-\mathrm{mg} / \mathrm{kg}$ (2 hours prior to surgery); I/R-Dc: 2-hours of ischemia and doxycycline 10-mg/kg and 2-hours of reperfusion. The groups were compared in terms of histological and biochemical features. A semi-quantitative histological assessment scoring system was used for the histological examination. Follicular cell degeneration, vascular congestion, haemorrhage, and inflammatory cell count were evaluated for histological analysis. For biochemical analysis of reperfusion injury, the body's total antioxidant status and total oxidant status were measured using a fully automatic method. The oxidative stress index was calculated by dividing total antioxidant status by total oxidant status.

RESULTS: In the sham group the ovaries were histologically normal. Oedema, vascular congestion, bleeding, leukocyte infiltration, and follicle degeneration were increased in other groups $(p<0.05)$. There was less leukocyte infiltration in the I/R-Dc group compared to I/R group. Other histological features were similar in these groups. Doxycycline increased the malondialdehyde, total antioxidant and total oxidant status levels in Sham-Dc and I/R-Dc groups. This increase was statistically significant between Sham and Sham-Dc groups. However, although there was an increase in the biochemical markers in the I/R-Dc group, this increase was not significant compared to I/R group.

CONCLUSION: Doxycycline treatment in ovarian torsion does not reduce I/R injury. Doxycycline may even increase reperfusion injury, according to biochemical findings.

Keywords: Doxycycline, Ovarian ischemia, Ovarian torsion, Rat model, Reperfusion injury Gynecol Obstet Reprod Med 2019;25(1):38-43

${ }^{1}$ Department of Obstetrics and Gynecology, University of Health Sciences Ankara Training and Research Hospital, Ankara

${ }^{2}$ Department of Obstetrics and Gynecology, University of Health Sciences, Zekai Tahir Burak Women's Health and Research Hospital, Ankara

${ }^{3}$ Department of Pathology, University of Health Sciences, Ankara Training and Research Hospital, Ankara

Address of Correspondence: Yetkin Karasu

Department of Obstetrics and Gynecology, University of Health

Sciences, Ankara Training and

Research Hospital, 06340 Altindag

Ankara, Turkey

dr.yetkinkarasu@gmail.com

Submitted for Publication:

10.02.2018

Accepted for Publication:

17.07.2018

ORCID IDs of the authors:

G.B.: 0000-0002-7527-2110, Y.K.: 0000-0002-0003-3502,

E.Y.: 0000-0002-1517-3823, C.T.: 0000-0002-5587-1757,

S.OS.: 0000-0002-1326-4246, A.OD.: 0000-0002-3298-4927,

O.U.: 0000-0003-3453-3852, T.G.: 0000-0002-7869-9662,

M.C.: 0000-0003-2910-288X

\begin{tabular}{|c|c|}
\hline Quick Response Code: & Access this article online \\
\cline { 2 - 2 } & Website: www.gorm.com.tr \\
\cline { 2 - 2 } & e- mail: info@gorm.com.tr \\
\hline
\end{tabular}

\section{Introduction}

Ovarian torsion is a partial or complete rotation of the ovary that disrupts its blood flow around the ligamentous connections. It is one of the most common gynaecological emergencies for all age groups $(1,2)$. Ischemia that develops secondary to impaired blood flow causes loss of ovarian reserve. Early diagnosis and surgical correction to restore blood flow are very important.

Reperfusion injury is the damage that occurs when blood flow is restored to under-oxygenated tissues. Microvascular dysfunction following reperfusion increases fluid filtration, leukocyte and plasma protein extravasation, and production of free oxygen radicals (3). Events occurring during reperfusion of ischemic tissue lead to increases in mediators such as TNF-alpha, interleukin-1, platelet activating factor, and nitric oxide (4).

How to cite this article: Bektas G. Karasu Y. Yurtcu E.Togrul C. Ozyer Sen S. Onder Dirican A. Uzunlar O. Gungor T. Caydere M. Effect of Doxycycline on Aseptic Reperfusion Injury in Ovarian Torsion. Gynecol Obstet Reprod Med $2019 ; 25(1): 38-43$ 
Doxycycline, a member of the tetracycline antibiotic family, inhibits mRNA transcription of the matrix metalloproteinases. It has anti-inflammatory and cytoprotective effects $(5,6)$. Its role in preventing reperfusion injury and reducing the number of mediators produced during reperfusion has been demonstrated previously in cardiac, renal, bowel and cerebral tissues $(4,7,8)$. Anti-apoptotic effects of doxycycline have also been found in mouse testes (9). Doxycycline reduces interleukin $1 \mathrm{~B}$ expression and inhibits matrix metalloproteinases $(10,11)$.

Oxidant status is the total burden that results from reactions at a molecular level and has a negative effect, whereas the protective response that develops to correspond to the prooxidant molecules is called the antioxidant effect. Total oxidant status (TOS) has been identified as the in vivo marker of a shift developing in an oxidative/antioxidative ratio in favour of the oxidative side. Total antioxidant status (TAS), on the other hand, reflects all total plasma antioxidant substrates. Oxidative stress index (OSI), an indicator of the degree of oxidative stress, is the percentage of the TOS level/TAS level ratio (12).

The aim of this study is to investigate whether doxycycline treatment reduces reperfusion injury in a rat model of ovarian torsion.

\section{Material and Method}

Approval for this study was obtained (0018/288) from the "Animal Experiments Local Ethics Committee" of Ankara Training and Research Hospital, Ministry of Health, Republic of Turkey. Thirty-six female Wistar albino rats were used (200-250 g). In this study, a similar method as in the studies of Yurtcu et al., who investigated the efficacy of vardenafil in ovarian torsion, was applied. (13). The animals were divided into five groups. The animals in the first group formed the sham group (Sham). Only laparotomy was performed on this group. The rats in the second group were laparotomized and the ovaries were left in torsion for 2 hours (I). In the third group, 2 hours of torsion and 2 hours of reperfusion were performed (I/R). In the fourth group, sham operation was performed following the application of intraperitoneal doxycycline $(10 \mathrm{mg} / \mathrm{kg})$ (Sham-Dc). In the fifth group, 2 hours of ischemia and doxycycline therapy $(10 \mathrm{mg} / \mathrm{kg})$ was followed by 2 hours of reperfusion (doxycycline treatment was started at the 1 st hour of the ischemic period) (I/R-Dc). Doxycycline (10 $\mathrm{mg} / \mathrm{kg}$; Monodoks ${ }^{\circledR} 100 \mathrm{mg}$ capsules; Deva) was administered intraperitoneal (i.p.) after being dissolved in $0.9 \% \mathrm{NaCl}$. Rats were anesthetized using intraperitoneal ketamine $(40 \mathrm{mg} / \mathrm{kg}$; $\operatorname{Ketalar}^{\circledR}$ Flacons $50 \mathrm{mg} / \mathrm{mL}$; Pfizer) and xylazine $(10 \mathrm{mg} / \mathrm{kg}$; Xylazin $^{\circledR} 20 \mathrm{mg} / \mathrm{mL}$ Flacons; Kepro). For laparotomy, a 2centimeter midline incision was made. Right adnexa was ligated bilaterally using 4/0 vicryl and left ischemic for 2 hours. After completion of the procedure in all groups, the right ovaries were removed and divided into two equal parts for biochemical and histopathological examination.
The tissues were stored at $-80^{\circ} \mathrm{C}$ until analysis. Tissue investigations were performed after thawing the samples to room temperature. The tissue samples were weighed on a scale with 0.001 grams sensitivity. The samples were diluted and homogenized using a mechanical homogenizer. The samples were then centrifuged at $3000 \mathrm{rpm}$ for 5 minutes, after which the supernatant was collected and the sample calorimetrically analysed using the "Rel Assay E" auto-analyser, as detailed below. "Rel Assay" kits were used for the analysis. The body's total antioxidant status (TAS) and total oxidant status (TOS) were measured using a fully automatic method developed by Ozcan Erel. The Oxidative Stress Index (OSI) was calculated by dividing TAS by TOS (14). The TOS was analysed using an automated calorimetric method, in which the oxidants present in the sample oxidize ferrous ion-o-dianisidine complexes to ferric ion. The ferric ions form a coloured complex with xylenol orange in the acidic medium, and the TOS levels determined spectrophotometrically $\left(\mu \mathrm{molH}_{2} \mathrm{O}_{2}\right.$ Equiv/L). The total antioxidant level (TAS) was measured using the automated method developed by Ozcan Erel that is based on the whitening of the characteristic blue-green colour of a stable radical cation 2,2'-Azinobis (3-ethylbenzothiazoline-6-sulfonic acid) (ABTS) by antioxidants (12). TAS levels were measured using with TAS assay kit (Rel Assay Diagnostic, Gaziantep, Turkey), providing an error margin of less than 3\% (mmoL Trolox equivalent $/ \mathrm{L})$. The malondialdehyde (MDA) assay is based on the reaction of MDA with thiobarbituric acid (TBA) at a reaction temperature of $90-100{ }^{\circ} \mathrm{C}$. The reaction was carried out at $90^{\circ} \mathrm{C}$ and $\mathrm{pH}$ : 2-3 for $15 \mathrm{~min}$ utes. Absorbance was measured at $532 \mathrm{~nm}$. The amount of MDA in the sample was calculated from the measurement graph drawn using 1.1.3.3 - tetramethoxypropane as a standard and is expressed as nmoL/mg protein.

A semi-quantitative histological assessment scoring system was used for the histological examination. Follicular cell degeneration, vascular congestion, haemorrhage, and inflammatory cells were evaluated. Each criterion was scored between 0 and 3 ( 0 : none; 1 : mild; 2: moderate; 3 : severe).

Statistical analyses were performed using SPSS 17.0 for Windows (SPSS, Inc., Chicago, Illinois). The results were analysed with a Kruskal-Wallis test for TAS, TOS, OSI and MDA. A chi-square test was used for histological data. $P<$ 0.05 was considered statistically significant.

\section{Results}

A total of 35 rats were used. One rat in the I/R group died during induction of anaesthesia. In the general histologic examination, ovaries were normal in the Sham group but oedema, vascular congestion, haemorrhage, PMNL infiltration and follicle degeneration were increased in the other groups $(p<0.05)$ (Table 1). Oedema, vascular congestion, haemorrhage, PMNL infiltration and follicular degeneration were increased in the ischemia and $I / R$ groups. Comparing the $I / R$ 
and I/R-Dc groups, PMNL infiltration was found to be less in the I/R-Dc group $(p=0.005)$. There were no differences between the two groups in terms of other histological features $(p>0.05)$ (Figure 1).

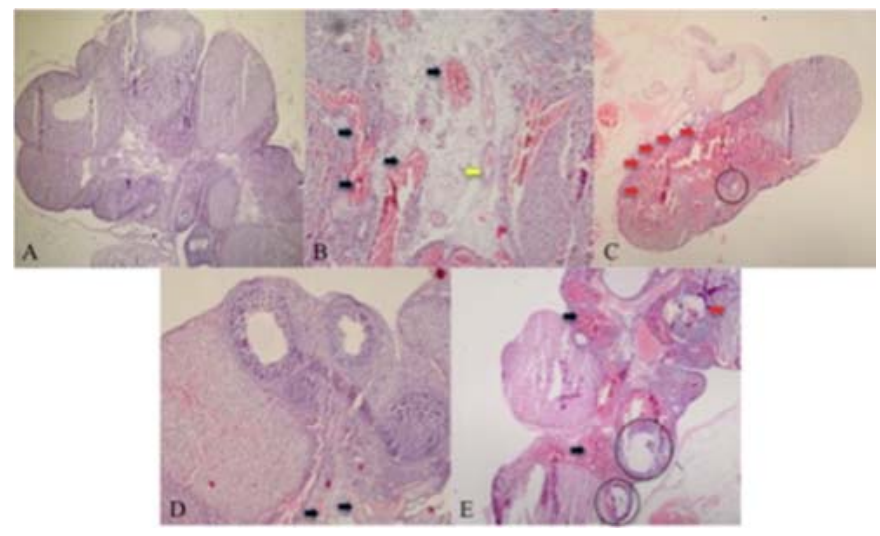

Figure 1: Histological features of the groups

A: Normal histology of ovarian tissue (Sham). B: Ischemia group. Ovarian haemorrhage (black arrow), important inflammation and oedema (yellow arrow). C: Ischemia/reperfusion group. Dense intrafollicular degeneration (red arrow). Follicular degeneration (black circle). D: Sham/ Doxycycline group. Mild vascular congestion (black arrow). E: Ischemia/ Reperfusion/Doxycycline group. Vascular congestion (black arrow). Intrafollicular haemorrhage (red arrow). Follicular degeneration (black circle).
When the sham and sham-Dc groups were compared, doxycycline was found to increase MDA, TAS and TOS levels and these changes were statistically significant $(p=0.004$, $p=0.001, p=0.001$, respectively) (Table 2). Similarly, MDA, TAS and TOS levels were elevated in rats given doxycycline (I/R-Dc) compared to I/R alone but this was not statistically significant (Figure 2-3).



Figure 2: The effect of doxycycline on MDA, TAS, TOS levels in ovarian tissues.

Sham: Sham group: I: Ischemia group, I/R: Ischemia and reperfusion group, Sham-Dc: Sham and doxycycline group, I/R-Dc: Ischemiareperfusion and doxycycline group, MDA: Malondialdehyde, TAS: Total antioxidant status, TOS: Total oxidant status, I/R: Ischemia/ Reperfusion, Dc: Doxycycline

Table 1: Comparison of histological findings

\begin{tabular}{|c|c|c|c|c|c|c|}
\hline & Sham & I & $\mathrm{I} / \mathrm{R}$ & Sham-Dc & I/R-Dc & $p$ value* \\
\hline \multicolumn{7}{|l|}{ Oedema } \\
\hline None & $7(100 \%)$ & $0(0 \%)$ & $1(16.7 \%)$ & $0(0 \%)$ & $0(0 \%)$ & \multirow[t]{3}{*}{$<0.001$} \\
\hline Grade I & $0(0 \%)$ & $6(85.7 \%)$ & $3(50 \%)$ & $7(\% 100)$ & $3(42.9 \%)$ & \\
\hline Grade II & $0(0 \%)$ & $1(14.3 \%$ & $2(33.3 \%)$ & $0(0 \%) 0$ & $4(57.1 \%)$ & \\
\hline \multicolumn{7}{|c|}{ Vascular Congestion } \\
\hline None & $3(42.9 \%)$ & $0(0 \%)$ & $0(0 \%) 1$ & $0(0 \%)$ & $0(0 \%)$ & \multirow[t]{4}{*}{0.001} \\
\hline Grade I & $4(57.1 \%)$ & $0(0 \%)$ & $(16.7 \%)$ & $4(57.1 \%)$ & $2(28.6 \%)$ & \\
\hline Grade II & $0(0 \%)$ & $3(42.9 \%)$ & $4(66.7 \%)$ & $3(42.9 \%)$ & $5(71.4 \%)$ & \\
\hline Grade III & $0(0 \%)$ & $4(57.1 \%)$ & $1(16.7 \%)$ & $0(0 \%)$ & $0(0 \%)$ & \\
\hline \multicolumn{7}{|c|}{ Haemorrhage } \\
\hline None & $6(85.7 \%)$ & $0(0 \%)$ & $0(0 \%)$ & $7(100 \%)$ & $0(0 \%)$ & \multirow[t]{4}{*}{$<0.001$} \\
\hline Grade I & $1(14.3 \%)$ & $2(28.6 \%)$ & $2(33.3 \%)$ & $0(0 \%)$ & $4(57.1 \%)$ & \\
\hline Grade II & $0(0 \%)$ & $1(14.3 \%)$ & $2(33.3 \%)$ & $0(0 \%)$ & $3(42.9 \%)$ & \\
\hline Grade III & $0(0 \%)$ & $4(57.1 \%)$ & $2(33.3 \%)$ & $0(0 \%)$ & $0(0 \%)$ & \\
\hline \multicolumn{7}{|l|}{ PMNL } \\
\hline None & $4(57.1 \%)$ & $1(14.3 \%)$ & $1(16.7 \%)$ & $4(57.1 \%)$ & $7(100 \%)$ & \multirow[t]{3}{*}{0.019} \\
\hline Grade I & $2(28.6 \%)$ & $6(85.7 \%)$ & $5(83.3 \%)$ & $342.9 \%$ & $0(0 \%)$ & \\
\hline Grade II & $1(14.3 \%)$ & $0(0 \%)$ & $0(0 \%)$ & $0(0 \%)$ & $0(0 \%)$ & \\
\hline \multicolumn{7}{|c|}{ Follicle Degeneration } \\
\hline None & $7(100 \%)$ & $0(0 \%)$ & $2(33.3 \%)$ & $7(100 \%)$ & $2(28.6 \%)$ & \multirow[t]{4}{*}{$<0.001$} \\
\hline Grade I & $0(0 \%)$ & $3(42.9 \%)$ & $1(16.7 \%)$ & $0(0 \%)$ & $5(71.4 \%)$ & \\
\hline Grade II & $0(0 \%)$ & $2(28.6 \%)$ & $3(50 \%)$ & $0(0 \%)$ & $0(0 \%)$ & \\
\hline Grade III & $0(0 \%)$ & $2(28.6 \%)$ & $0(0 \%)$ & $0(0 \%)$ & $0(0 \%)$ & \\
\hline
\end{tabular}

${ }^{*}$ Chi-square test. Sham: Sham group, I: Ischemia group, I/R: Ischemia and reperfusion group, Sham-Dc: Sham and doxycycline group; I/R-Dc: Ischemia-reperfusion and doxycycline group 
Table 2: Comparison of the biochemical results for groups

\begin{tabular}{lrrrrrr}
\hline & \multicolumn{1}{c}{ Sham } & \multicolumn{1}{c}{ I } & \multicolumn{1}{c}{ I/R } & Sham-Dc & I/R-Dc & $p$ value* \\
\hline MDA $(\mu \mathrm{moL} / \mathrm{g})$ & $20.52 \pm 5.22$ & $37.39 \pm 8.35$ & $29.31 \pm 7.11$ & $33.25 \pm 6.88$ & $38.44 \pm 6.80$ & 0.002 \\
TAS $\left(\mathrm{mmoL} \mathrm{H} \mathrm{O}_{2}\right.$ & $0.59 \pm 0.13$ & $0.70 \pm 0.28$ & $0.59 \pm 0.19$ & $0.86 \pm 0.17$ & $0.75 \pm 0.28$ & 0.088 \\
Equiv./L) & & & & & & \\
TOS $\left(\mu \mathrm{moLH}{ }_{2} \mathrm{O}_{2}\right.$ & $5.71 \pm 1.76$ & $14.54 \pm 6.93$ & $12.56 \pm 5.48$ & $11.01 \pm 2.31$ & $15.65 \pm 5.48$ & 0.007 \\
Equiv./L) & $10.17 \pm 3.61$ & $19.87 \pm 3.49$ & $20.66 \pm 4.30$ & $13.01 \pm 3.10$ & $21.43 \pm 4.70$ & $<0.001$ \\
OSI & & & & & & \\
\hline
\end{tabular}

*Kruskal Wallis Test. Sham: Sham group, I: Ischemia group, I/R: Ischemia and reperfusion group, Sham-Dc: Sham and doxycycline group, I/R-Dc: Ischemia-reperfusion and doxycycline group, OSI: Oxidative stress index

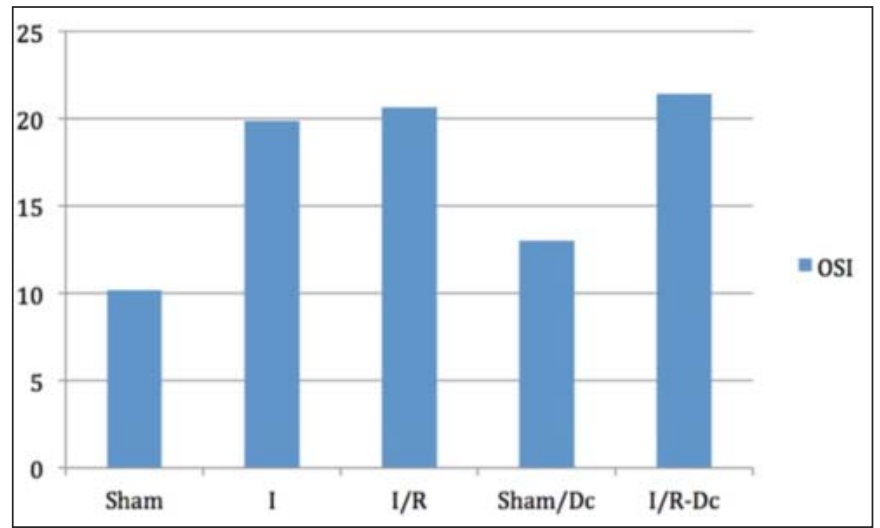

Figure 3: OSI in the groups

Sham: Sham group,; I: Ischemia group, I/R: Ischemia and reperfusion group, Sham-Dc: Sham and doxycycline group, I/R-Dc: Ischemiareperfusion and doxycycline group, OSI: Oxidative stress index

\section{Discussion}

In our study, the use of doxycycline in the ovarian torsion rat model did not reduce reperfusion injury. On the contrary, doxycycline was found to increase levels of MDA and TOS levels in the ischemia group compared to the sham group. This was also true for the I/R groups. MDA and TOS levels were higher in doxycycline-treated rats than doxycycline-free $\mathrm{I} / \mathrm{R}$ group. Histological examination showed no significant effect of doxycycline on ovarian tissue, except for a reduction in PMNL infiltration.

As far as we know, our study is the first that investigates whether doxycycline reduces reperfusion injury in ovarian torsion or not. Although there were studies reporting that doxycycline reduces reperfusion injury in liver, kidney, bowel, heart and brain tissues; there has been no such report for ovarian torsion. For this reason, we think it is important to see that doxycycline may increase the markers of reperfusion injury in our study.

Our study is important in comparing sham, ischemia and $\mathrm{I} / \mathrm{R}$ groups with a rat model, but since a fixed doxycycline dose was used; different doses of doxycycline have not been tested. The dose of doxycycline used was determined by taking into account previous studies. The most important limitation of our study is that, different doses of doxycycline were not tested.
Another limitation of the study is that, the reproductive potential of the animals following the tests was not evaluated. Undoubtedly, any treatment for ovarian torsion aims to reduce ovarian damage and to preserve hormonal competence and fertility. Therefore, it is important to evaluate the long-term effects of treatment on issues such as fertility and menopausal status. This is, however, difficult to do in an animal model.

In ovarian torsion, early surgical treatment is critical to prevent damage due to ischemia. However, this treatment, which improves blood flow, causes reperfusion injury in tissues that have been left without oxygen for a while. After an ischemic period, regaining the circulation can lead to inflammation and oxidative damage. This presents a problem for the ovaries and the germ cell population they contain because there is no way to replace the lost ovarian reserve.

Several studies in the literature have investigated ways to reduce reperfusion injury following ovarian torsion and surgical treatment. Many substances have been found to reduce reperfusion injury after surgical treatment. These include agents that increase blood flow, such as iloprost, as well as radical cleansing agents such as edaravone $(15,16)$. Some substances act directly by inactivating reactive oxygen radicals $(17,18)$. Almost all of these studies were done in animal models. To the best of our knowledge, no previous study has studied the efficacy of doxycycline in ovarian torsion.

Many studies have shown that doxycycline can reduce reperfusion injury in various tissues. For example, Ihtiyar et al. found that $10-\mathrm{mg} / \mathrm{kg}$ doxycycline reduced pro-inflammatory cytokine levels and metalloproteinase-2 (MMP-2) activity while increasing tissue inhibitor metalloproteinase-1 (TIMP-1) levels in a rat renal torsion model. The authors thus concluded that doxycycline prevents apoptosis due to $\mathrm{I} / \mathrm{R}$ injury in renal tissue (4). Yasar et al. demonstrated a similar effect in intestinal tissue (19). Schulze et al. observed that doxycycline reduces MMP-2 activity in coronary artery disease patients (7).

Doxycycline may protect the cell from I/R damage by the following ways. It reduces apoptosis by decreasing mitochondrial $\mathrm{Ca}^{2+}$ uptake and mitochondrial permeability transition (20). It also reduces production of reactive oxygen radicals and induces the antioxidant and cytoprotective pathways such 
as nuclear factor erythroid 2-related factor 2 (Nrf2) (21). Doxycycline may decrease the production and activity of matrix metalloproteinases and it reduces effect and amount of pro-inflammatory cytokines (IL-1B, IL-6, TNF-A) $(8,19)$.

While these results lead to the suggestion that doxycycline may reduce reperfusion injury following treatment for ovarian torsion, such an effect was not seen in our study. Each tissue can respond differently to ischaemia and reperfusion. The tissue's response to such injury depends on the severity of the ischemia and the tissue's ability to heal itself. A more severe response to ischemia may be expected in tissues with high metabolic demand, such as in the brain, where the glucose/ glycogen deposits are very low $(22,23)$. In the skeleton, on the other hand, the response to ischemia and reperfusion injury is more benign. Another factor that determines the tissue's response is its protective antioxidant mechanisms. Reduced levels of antioxidants such as superoxide dismutase, catalase, and glutathione peroxidase may exacerbate ischemia/reperfusion injury. Its unique features may make ovarian tissue more vulnerable to ischemia and reperfusion injury. This may be the reason why the anticipated effect of doxycycline was not observed.

Although there are studies in the literature demonstrating that tetracycline reduces I/R damage, there are studies reporting that they have different effects. Tetracycline can affect metabolic gene expression in human cells. In vitro studies have shown that tetracycline can affect glycolytic and oxidative processes in different cell lines (dose-dependent action) and reduce cell proliferation (24). It may have also anti-inflammatory and immunosuppressive effects (25). It has also been reported that tetracycline can disrupt mitochondrial function (26). With these effects, tetracycline can cause toxic effects in cells. Due to all these, doxycycline might not have reduced the reperfusion damage as expected.

In conclusion, in our study, doxycycline was not found to reduce $\mathrm{I} / \mathrm{R}$ injury in ovarian torsion. Conversely, oxidative damage markers such as MDA and TOS were increased in cases treated with doxycycline. Thus, for ovarian torsion cases, the use of doxycycline does not seem to have a protective effect. Further studies are necessary to see whether these results are supported.

\section{: Acknowledgement: None}

Conflict of interest: The authors declare no conflicts of interest.

Funding: None

\section{References}

1. McWilliams GD, Hill MJ, Dietrich CS $3^{\text {rd }}$. Gynecologic emergencies. Surg Clin North Am. 2008;88(2):265-83.

2. Tayyar AT, Ozkaya E, Abide Yayla C, Sentürk MB, Selcuk S, Polat M, et al. Evaluation of Complete Blood
Count Parameters to Predict Ovarian Torsion in Women with Adnexal Mass. Gynecol Obstet Reprod Med. 2017; 23(2):89-93.

3. Carden DL, Granger DN. Pathophysiology of ischaemiareperfusion injury. J Pathol. 2000;190(3):255-66.

4. Ihtiyar E, Yasar NF, Erkasap N, Koken T, Tosun M, Oner $\mathrm{S}$, et al. Effects of doxycycline on renal ischemia reperfusion injury induced by abdominal compartment syndrome. J Surg Res. 2011;167(1):113-20.

5. Jantzie LL, Cheung PY, Todd KG. Doxycycline reduces cleaved caspase- 3 and microglial activation in an animal model of neonatal hypoxia-ischemia. J Cereb Blood Flow Metab. 2005;25(3):314-24.

6. Krakauer T, Buckley M. Doxycycline is anti-inflammatory and inhibits staphylococcal exotoxin-induced cytokines and chemokines. Antimicrob Agents Chemother. 2003;47(11):3630-3.

7. Schulze CJ, Castro MM, Kandasamy AD, Cena J, Bryden $\mathrm{C}$, Wang $\mathrm{SH}$, et al. Doxycycline reduces cardiac matrix metalloproteinase-2 activity but does not ameliorate myocardial dysfunction during reperfusion in coronary artery bypass patients undergoing cardiopulmonary bypass. Crit Care Med. 2013;41(11):2512-20.

8. Wang Z, Xue Y, Jiao H, Liu Y, Wang P. Doxycycline-mediated protective effect against focal cerebral ischemiareperfusion injury through the modulation of tight junctions and PKCdelta signaling in rats. J Mol Neurosci. 2012;47(1):89-100.

9. Yeh YC, Lai HC, Ting CT, Lee WL, Wang LC, Wang KY, et al. Protection by doxycycline against doxorubicin-induced oxidative stress and apoptosis in mouse testes. Biochem Pharmacol. 2007;74(7):969-80.

10. Golub LM, Lee HM, Ryan ME, Giannobile WV, Payne J, Sorsa T. Tetracyclines inhibit connective tissue breakdown by multiple non-antimicrobial mechanisms. Adv Dent Res. 1998;12(2):12-26.

11. Xiao QG, Liu ZG, Zhang M, Zheng JL, Zhang ZH, Luo $\mathrm{LH}$, et al. [Effect of doxycycline on inflammation-related cytokines and apoptosis in human conjunctival epithelial cells]. Zhonghua Yan Ke Za Zhi. 2005;41(9):842-6.

12. Erel O. A novel automated method to measure total antioxidant response against potent free radical reactions. Clin Biochem. 2004;37(2):112-9.

13. Yurtcu E, Togrul C, Ozyer S, Uzunlar O, Karatas YH, Seckin KD, et al. Dose dependent protective effects of vardenafil on ischemia-reperfusion injury with biochemical and histopathologic evaluation in rat ovary. J Pediatr Surg. 2015;50(7):1205-9.

14. Erel O. A new automated colorimetric method for measuring total oxidant status. Clin Biochem. 2005;38(12):110311 .

15. Ozat M, Gungor T, Barun S, Demirogullari B, Sokmensuer LK, Gulbahar O, et al. The effects of iloprost, 
a prostacyclin analogue, in experimental ischaemia/reperfusion injury in rat ovaries. Exp Toxicol Pathol. 2009;61(5):519-27.

16. Kara M, Daglioglu YK, Kuyucu Y, Tuli A, Tap O. The effect of edaravone on ischemia-reperfusion injury in rat ovary. Eur J Obstet Gynecol Reprod Biol. 2012;162 (2):197-202.

17. Luck MR, Jeyaseelan I, Scholes RA. Ascorbic acid and fertility. Biol Reprod. 1995;52(2):262-6.

18. Zreik TG, Kodaman PH, Jones EE, Olive DL, Behrman H. Identification and characterization of an ascorbic acid transporter in human granulosa-lutein cells. Mol Hum Reprod. 1999;5(4):299-302.

19. Fatih Yasar N, Ozdemir R, Ihtiyar E, Erkasap N, Koken T, Tosun M, et al. Effects of doxycycline on intestinal ischemia reperfusion injury induced by abdominal compartment syndrome in a rat model. Curr Ther Res Clin Exp. 2010;71(3):186-98.

20. Schwartz J, Holmuhamedov E, Zhang X, Lovelace GL, Smith CD, Lemasters JJ. Minocycline and doxycycline, but not other tetracycline-derived compounds, protect liver cells from chemical hypoxia and ischemia/reperfu- sion injury by inhibition of the mitochondrial calcium uniporter. Toxicol Appl Pharmacol. 2013;273(1):172-9.

21. Malik YS, Sheikh MA, Zhu X. Doxycycline can stimulate cytoprotection in neural stem cells with oxygen-glucose deprivation-reoxygenation injury: a potential approach to enhance effectiveness of cell transplantation therapy. Biochem Biophys Res Commun. 2013;432(2):355-8.

22. Kristián T. Metabolic stages, mitochondria and calcium in hypoxic/ischemic brain damage. Cell Calcium. 2004; 36(3-4):221-33.

23. Lee JM, Grabb MC, Zipfel GJ, Choi DW. Brain tissue responses to ischemia. J Clin Invest. 2000;106(6):723-31.

24. Ahler E, Sullivan WJ, Cass A, Braas D, York AG, Bensinger SJ, et al. Doxycycline alters metabolism and proliferation of human cell lines. PLoS One. 2013; 8(5):e64561.

25. Roberts MC. Tetracycline therapy: update. Clin Infect Dis. 2003;36(4):462-7.

26. Chatzispyrou IA, Held NM, Mouchiroud L, Auwerx J, Houtkooper RH. Tetracycline antibiotics impair mitochondrial function and its experimental use confounds research. Cancer Res. 2015;75(21):4446-9. 